\title{
Multicomponent Crystals with Two Fast Reorienting Constituents: Mutually Affected Rotation of DABCO and THF
}

\author{
Abraham Colin-Molina, ${ }^{\ddagger}$ Jessica Arcudia," Eliseo R. López-López, ${ }^{\ddagger}$ Marcus J. Jellen, ${ }^{\S}$ \\ Ma. Carmen García-González, ${ }^{\ddagger}$ Gabriel Merino, ${ }^{\text {I* }}$ and Braulio Rodríguez-Molina ${ }^{\ddagger *}$ \\ ‡Universidad Nacional Autónoma de México, Circuito Exterior, Ciudad Universitaria, 04510, Ciudad \\ de México, México. \\ ${ }^{\S}$ Department of Chemistry and Biochemistry, University of California, Los Angeles, 90095, California, \\ United States. \\ "Departamento de Física Aplicada, Centro de Investigación y de Estudios Avanzados, Unidad \\ Mérida. Km. 6 Antigua Carretera a Progreso, Apdo. Postal 73, Cordemex, 97310, Mérida, Yuc., \\ México.
}

\begin{abstract}
Here we report two new multicomponent supramolecular rotors ( 1 and $\mathbf{2}$ ) based on 2,7diphenyl-9H-carbazole stators and two rotators brought together by a non-innocent $\mathrm{C}-\mathrm{H} \cdots \mathrm{O}$ interaction. This hydrogen bond serves a dual function: it constitutes the rotational axis for THF and acts as a 'brake' for the motion of DABCO. Dynamic characterization of $\mathbf{1}$ by VT ${ }^{2} \mathrm{H}$ NMR experiments supports that DABCO rotates about its supramolecular axis with jumps of 120 o (3-fold) and a $k_{\text {rot }}>10 \mathrm{MHz}$ at $300 \mathrm{~K}$, but interestingly, this motion is reduced to $5 \mathrm{MHz}$ at $150 \mathrm{~K}$. Concurrently, the THF experiences an atypical motion on an axis that is perpendicular to that of DABCO, experiencing 180 0 jumps in the fast regime even at $175 \mathrm{~K} .{ }^{1} \mathrm{H}\left(\mathrm{T}_{1}\right)$ relaxometry studies show that DABCO has an $E a=3.5 \mathrm{kcal} / \mathrm{mol}$, while for THF, an $E a=2.3 \mathrm{kcal} / \mathrm{mol}$ was computed by periodic DFT. Fast and organized mobility of two constituents within crystals is extremely infrequent and could have strong implications for future applications of multicomponent solids with regulated dynamics.
\end{abstract}

In recent years, supramolecular chemistry has undergone colossal progress due to the construction of new and complex systems with notable applications in drug delivery ${ }^{1}$ or separation and storage of vapors and gases. ${ }^{2}$ From work on cryptophanes ${ }^{3}$ and cryptands, ${ }^{4}$ to more recent developments on cages $^{5}$ and capsules, $^{6}$ it can be seen that directional interactions, such as hydrogen bonds, are used to assemble frameworks of increasing complexity, more specifically on molecular rotors. ${ }^{7}$ These molecules exhibit machine-like segmental displacements, combining long-range order (periodicity) and a fast-rotating portions (mobility). One of the motivations for creating such systems is that the collective motion could enable the construction of artificial muscles, ${ }^{8}$ and adaptronic systems, ${ }^{9}$ being the internal motion the key to the crystal's responsiveness. ${ }^{10}$

One way to obtain these artificial synthetic machines is to devise amphidynamic crystals through the synthesis of molecules with dumbbell-shapes. This design incorporates a rotary component (rotator) in between large groups that create the crystal lattice (stator). ${ }^{11}$ Following this blueprint, our group recently reported a series of supramolecular rotors by placing $\mathrm{DABCO}$ between carbazole derivatives. ${ }^{12}$ Other groups have followed different strategies, such as the use of porous crystals as the framework that favors the inclusion of linear rotary components. ${ }^{13}$ In the realm of crystal engineering field, realizing larger complexity in the solid-state is very interesting, ${ }^{14}$ particularly in the development of multicomponent solids for several applications, including, but not limited to, active pharmaceutical ingredients $(\mathrm{APIs})^{15}$, sensors ${ }^{16}$ and dielectrics. ${ }^{17}$

Herein we describe the synthesis and structural characterization of two multicomponent supramolecular rotors $\mathbf{1}$ and $\mathbf{2}$ using two angularly extended carbazole stators $\mathbf{7}$ and $\mathbf{8}$, which were designed to facilitate motion and promote new intermolecular interactions within the crystal. DABCO (1,4-diazabicyclo[2.2.2] octane) was chosen as the rotator because it is a base known to form hydrogen bonds with this type of stators and its plastic 
crystal nature is favorable in the construction of soft materials. ${ }^{18}$ The conceived stators were constructed to create enough space for DABCO and allow the occlusion of another component, in this case tetrahydrofuran (THF) molecule (Figure 1). Combining solid-state NMR, relaxometry studies, and density functional theory (DFT) computations, we conclude that both guests rotate over preferential axes at very high frequencies, contrary to the most commonly observed behavior where the presence of one component completely restricts the mobility of the other. ${ }^{19}$

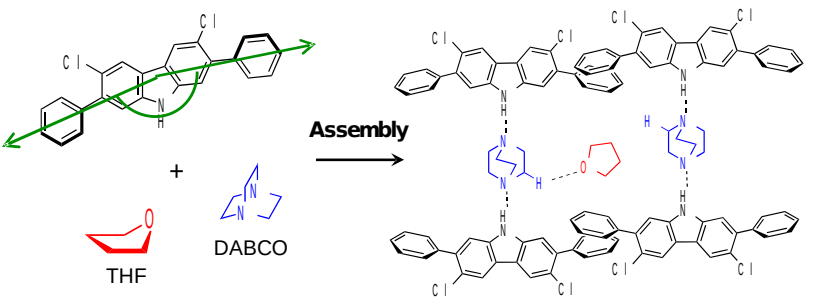

Figure 1. Representation of the multicomponent rotor $\mathbf{1}$ studied in this work.

To include the aromatic substituents in the positions 2 and 7 of the carbazole framework, a three-step route was followed (see Supporting Information). In this way, the stators 2,7-diphenyl3,6-dichloro-9H-carbazole 7 and 2,7-bis(2,3difluorophenyl)-3,6-dichloro-9H-carbazole 8 were obtained. It is interesting to note that the addition of chlorine atoms to the carbazole frameworks increases the solubility of the stators and forces a twisted conformation of the appended aromatic rings. The desired angularity of the stators $\mathbf{7}$ and 8 was corroborated from the corresponding X-ray structures. The appended rings in $\mathbf{7}$ form an angle of 170ㅇ, while in 8 the angle is 1650 (Figure S1-S2). The subsequent cocrystallization of $\mathbf{7}$ and 8 with DABCO rendered the 2:1 triad of $\mathbf{1}$. Unfortunately, no single crystals of $\mathbf{2}$ suitable for X-ray studies have been obtained so far.
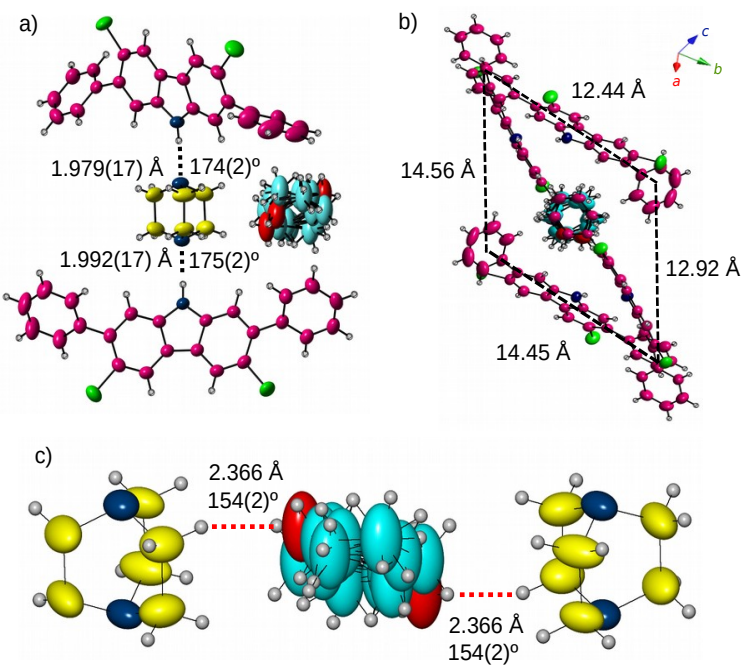

Figure 2. Structure of molecular rotor $\mathbf{1}$ at $298 \mathrm{~K}$. a) Asymmetric unit with the geometric parameters of the $\mathrm{H}$-bonds. b) Top-down view of the cavity formed by four stators around the THF. c) $\mathrm{C}-\mathrm{H} \cdots \mathrm{O}$ interactions of DABCO and the disordered THF (two-positions).

Rotor 1, collected at $298 \mathrm{~K}$, establishes strong $\mathrm{N}-\mathrm{H} \cdots \mathrm{N}$ hydrogen bonds between DABCO and the stators, with distances of 1.979 (17) and 1.992(17) $\AA$ and angles of 174(2)을 respectively (Figure $2 a$ ). The distances in the crystal between the nitrogen atoms of these hydrogen bonds are 2.85 and $2.87 \AA$, which are in excellent agreement with those computed by periodic DFT calculations $(2.81$ and $2.80 \AA)$. Interestingly, the cavity is large enough to facilitate the inclusion of a tetrahydrofuran (THF) molecule (Figure $2 \mathrm{~b}$ ). Note that there is a $\mathrm{C}-\mathrm{H} \cdots \mathrm{O}$ interaction between the two guests with an $\mathrm{H} \cdots \mathrm{O}$ distance of $2.366 \AA$ (Figure $2 \mathrm{c}$ ), which is considerably shorter than those found in other THF solvates $(2.62-2.71 \AA) .{ }^{20}$ The computed $\mathrm{CH}^{\cdots} \mathrm{O}$ distance $(2.38 \AA)$ is also in good agreement with the experimental data. The DABCO component is not disordered in $\mathbf{1}$, but the THF molecule occupies two positions, with its thermal ellipsoids suggesting a preferred vibrational or rotational mode above and below its molecular plane (Figure 2c).

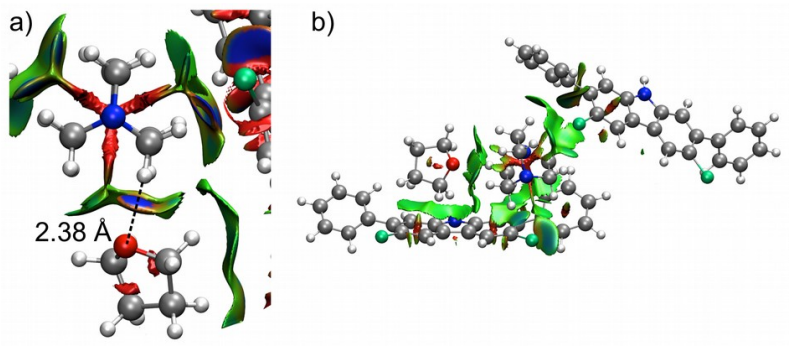

Figure 3. a) Non-covalent index ( $\mathrm{NCl}$ ) schematic representation between DABCO and THF, b) Neighboring interactions in $\mathbf{1}$.

Further structural insights were obtained using the non-covalent index $(\mathrm{NCl})$. According to this analysis, the DABCO component has three types of interactions. The first is with the THF via the C$\mathrm{H} \cdots \mathrm{O}$ interaction. The second set of contacts correspond to six $\mathrm{C}-\mathrm{H} \cdots \mathrm{Cl}$ interactions between $\mathrm{DABCO}$ and carbazoles. Finally, there are contacts between DABCO and the $\pi$-cloud of four terminal aromatic rings of the stators. Similarly, THF presents two types of interactions: $\mathrm{C}-\mathrm{H} \cdots \mathrm{O}$ contact with the DABCO and the interaction between methylenes and $\pi$-cloud of the two nearest chlorinated rings (Figure 3 ).

To prepare larger batches of $\mathbf{1}$ and $\mathbf{2}$ and subject them to solid-state NMR measurements, the Liquid-Assisted Grinding (LAG) method was used. ${ }^{21}$ In the case of $\mathbf{1}$, a comparison between calculated and experimental PXRD patterns confirmed that the THF solvate with the highest phase purity could be obtained within minutes (Figure S3-S4). Similarly, the ground mixture of 8 and DABCO produces a new solid with a unique diffraction pattern (Figure $\mathrm{S3}$ ). ${ }^{13} \mathrm{C}$ CPMAS 
experiments show distinct signals from the DABCO and THF molecules within the powdered solids, with changes in chemical shifts of the stator when compared to the starting materials, supporting the newly formed $\mathrm{N}-\mathrm{H} \cdots \mathrm{N}$ hydrogen bond (Figure S7).

Considering that the two guest molecules in $\mathbf{1}$ and $\mathbf{2}$ can be substituted by isotopically enriched DABCO or THF, deuterated samples were prepared using DABCO- $d_{10}$ or THF- $d_{8}$. The resulting solids were also analyzed by PXRD (Figure S5-S6) and ${ }^{13} \mathrm{C}$ CPMAS experiments (Figure S8-S9). The ${ }^{2} \mathrm{H}$ NMR spectrum of $\mathbf{1}$ shows that, at room temperature, the deuterated DABCO undergoes very fast jumps of 120 o along the supramolecular $\mathrm{N}-\mathrm{H} \cdots \mathrm{N}$ axis $\left(k_{\text {rot }}>10 \mathrm{MHz}\right)$, with a small isotropic contribution attributed to a minor amorphous component from the LAG synthesis (Figure 4, left). The ${ }^{2} \mathrm{H}$ line shape does not change in the 300-200 $\mathrm{K}$ range (Figure S10), but below this temperature, it starts to develop broader shoulders attributable to the gradual reduction of its rotational motion. At $150 \mathrm{~K}$, the trace agrees very well with a simulated line shape indicating a reduced rotational frequency of $5 \mathrm{MHz}$ (Figure 4, bottom left). The slowdown of this globular rotator is unusual, ${ }^{22}$ and suggests that its rotation may be affected by the presence of the neighboring THF. Considering this, it seems possible that such adjacency could also modify the motion of THF.

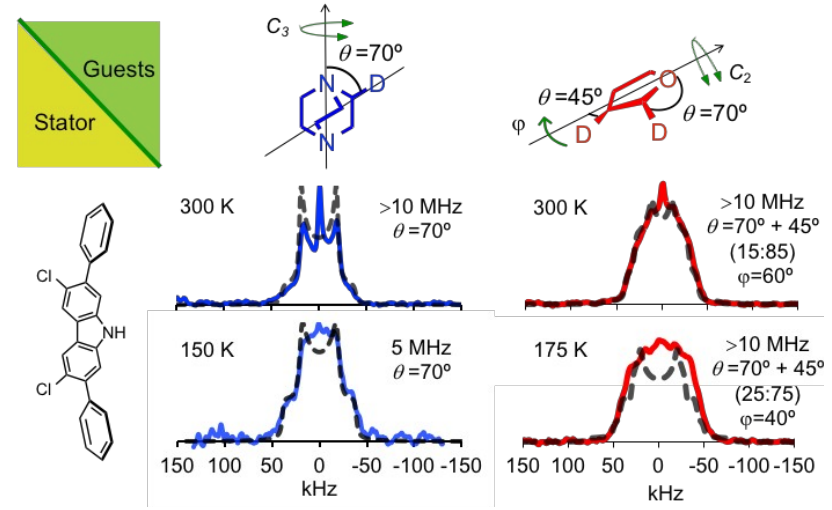

Figure 4. Experimental (solid lines) and simulated (dashed lines) ${ }^{2} \mathrm{H}$ NMR spectra for molecular rotor $\mathbf{1}$ between 150 and $300 \mathrm{~K}$.

To test this premise, the sample of $\mathbf{1}$ grown from deuterated THF was analyzed. The experiments show a deuterium line shape significantly broadened at room temperature and down to $175 \mathrm{~K}$ (Figure 4, right). These findings are unexpected because the motion of THF in numerous clathrate structures is extremely fast and occurs around multiple axes, producing a narrow deuterium signal (isotropic), even at temperatures as low as $125 \mathrm{~K}^{23}$ To reproduce the broad experimental spectra of $\mathbf{1}$, a dynamic model involving 180 jumps (2-fold) with large amplitude librations $(\phi)$ of 60 을 was proposed. In this case, the two types of deuterons contribute in a weighted manner to the spectra, probably due to the differences in its conformational mobility (Figure S11). The results clearly indicate that the THF rotation in $\mathbf{1}$ takes place around a preferred rotational axis: the intermolecular $\mathrm{C}-\mathrm{H}^{*}$ $\mathrm{O}$ interaction. In the case of rotor $\mathbf{2}$, the lack of structure and complexity of ${ }^{2} \mathrm{H}$ NMR spectra (Figure S12) prevented us from further studies of this rotor.
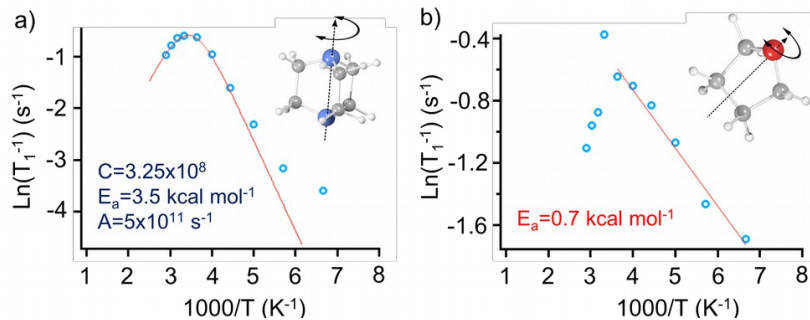

Figure 5. ${ }^{1} \mathrm{H} \mathrm{T}_{1}$ relaxation plots of $\mathrm{Ln}\left(1 / \mathrm{T}_{1}\right)$ vs $1000 /$ $\mathrm{T}$ of the protonated mobile components in rotor $\mathbf{1}$. a) Relaxation behavior or DABCO. b) Relaxation behavior of THF.

${ }^{1} \mathrm{H}$ spin-lattice relaxation experiments were carried out to study each mobile component independently in $\mathbf{1}$, taking advantage of the fact that the deuterated components of the samples do not contribute to the $\mathrm{T}_{1}$ (Figure S13-S14). It was possible to construct $\operatorname{Ln}\left(1 / T_{1}\right)$ vs $1000 / T$ plots for both rotating components (Figure 5). Note that the DABCO relaxation behavior reaches a minimum at about $270 \mathrm{~K}$, which occurs when $\tau_{c}$ coincides the Larmor frequency $(600 \mathrm{MHz})$. The fitting indicates that its rotational dynamics has an $E a=3.5 \mathrm{kcal} / \mathrm{mol}$ with a pre-exponential factor A of $5 \times 10^{11} \mathrm{~s}^{-1}$. The latter is one order of magnitude lower than the typical value reported for DABCO in other supramolecular rotors, suggesting a geometrically constrained transition state, ${ }^{24}$ probably due to the influence of the adjacent THF. In the relaxation studies for THF, no $\mathrm{T}_{1}$ minimum is found, indicating that fast molecular reorganization occurs at frequencies above $600 \mathrm{MHz}$ at $150 \mathrm{~K}$. The discontinuity around $330 \mathrm{~K}$ is associated with the desolvation process of $\mathbf{1}$. From the slope of the data, an activation energy of $0.7 \mathrm{kcal} / \mathrm{mol}$ is obtained, which is consistent with the rotational activation energy between $0.5-0.9 \mathrm{kcal} / \mathrm{mol}$ for THF in other reported crystal structures. ${ }^{25}$
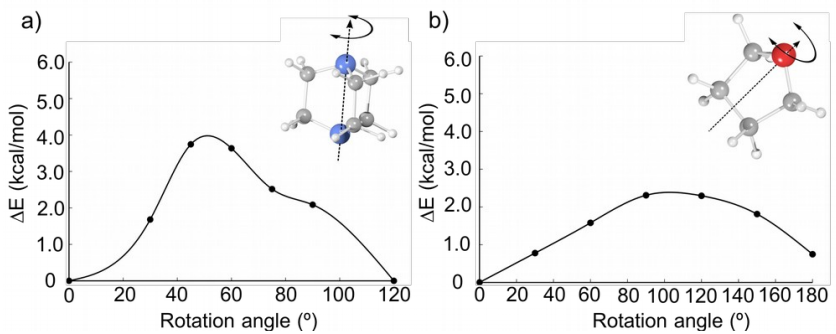

Figure 6. Computed rotational potential through periodic DFT, a) The barrier of DABCO $E a=4.0$ $\mathrm{kcal} / \mathrm{mol}, \mathrm{b})$ The barrier of THF Ea $=2.4 \mathrm{kcal} / \mathrm{mol}$. 
To better understand the experimental barriers, we calculated the rotational barriers within the crystal using a PBE functional, including scattering by the Grimme approximation. These periodic calculations were performed in VASP. Figure $6 a$ shows that the calculated rotational barrier for DABCO is $4.0 \mathrm{kcal} / \mathrm{mol}$, in excellent agreement with experimental value from NMR measurements. The computed barrier is not symmetrical, resulting from the four chlorine atoms surrounding this fragment with different distances. The transition state is at $51^{\circ}$, and a shoulder appears at $81^{\circ}$. On the other hand, the calculated rotation potential for THF (2.4 $\mathrm{kcal} / \mathrm{mol}$ ) is flatter with a maximum at 100 , and the rotation occurs on the axis defined by the $\mathrm{CH}^{\cdots} \mathrm{O}$ interaction (Figure $6 \mathrm{~b}$ ).

So, what causes the $0.7 \mathrm{kcal} / \mathrm{mol}$ experimental barrier? Both X-ray data and DFT computations indicate that THF adopts an enveloped conformation $\left(C_{s}\right)$. Note that pure THF crystallizes in a $C_{2}$ symmetry in the centrosymmetric space group C2/c with the oxygen atom located on the $\mathrm{C}_{2}$ crystallographic polar axis. ${ }^{26}$ However, THF undergoes several conformational changes both in the gas phase and in the crystal. The energy difference between the $C_{s}$ and $C_{2}$ conformers in the gas phase does not exceed $0.2 \mathrm{kcal} / \mathrm{mol}$. In our case, the $0.7 \mathrm{kcal} / \mathrm{mol}$ of the occluded THF could come from a combination of rotational and conformational modes restricted within the crystalline framework.

In summary, we developed two new multicomponent rotors $\mathbf{1}$ and $\mathbf{2}$ based on angular carbazole stators containing reorienting DABCO and THF molecules. Using a selective deuteration approach, small activation barriers for the rotation of each component of rotor $\mathbf{1}$ are elucidated through solid-state ${ }^{2} \mathrm{H}$ NMR with DABCO showing an $E a=3.5 \quad \mathrm{kcal} / \mathrm{mol}$. Complementary $T_{1}$ relaxation experiments yield an $E a=0.7 \mathrm{kcal} / \mathrm{mol}$, associated with rotational and conformational rearrangements of the THF molecule. The barriers obtained using periodic DFT computations are in good agreement with the experimental values. Considering all the results, we postulate that the slowdown of DABCO at low temperature is caused by THF, which acts as a molecular brake. Simultaneously, the interaction between the molecules creates a preferential axis for the 2-fold motion of THF, for which periodic DFT computations indicate a barrier of $2.4 \mathrm{kcal} / \mathrm{mol}$. Our results indicate that it is possible to obtain multicomponent crystals with two or more constituents reorienting at extremely fast frequencies, which could have strong implications on the optical and/or mechanical properties of multicomponent crystals.

\section{ASSOCIATED CONTENT}

\section{Supporting Information}

The Supporting Information is available free of charge on the ACS Publications website.

Experimental details (synthesis, ${ }^{1} \mathrm{H}$ and ${ }^{13} \mathrm{C}$ NMR solution data, single-crystal X-ray diffraction analysis), table with crystallographic parameters, variable-temperature ${ }^{1} \mathrm{H} \quad \mathrm{T}_{1}$ saturation-recovery experiments and variable-temperature spin-echo ${ }^{2} \mathrm{H}$ NMR measurements. Supporting Information is available free of charge on the ACS Publications website. CCDC numbers: 2069390-2069392.

\section{AUTHOR INFORMATION}

\section{Corresponding Author}

*gmerino@cinvestav.mx

*brodriguez@iquimica.unam.mx

\section{ACKNOWLEDGMENT}

A.C.M. and J.A. thank CONACYT for PhD fellowships (576483 and 859351). This project was supported by PAPIIT (Grant IN209119) and CONACYT (A1-S32820). We acknowledge the UCLA Department of Chemistry and Biochemistry (NSF DMR-1700471 and MRI-1532232) and the assistance from R. A. Toscano, B. Quiroz, M. A. Peña-González, E. HuertaSalazar. We thank UNAM's BGSI node for solid-state NMR measurements and MIZTLI for computer time (LANCAD-UNAM-DGTIC-392).

\section{REFERENCES}

1. Wankar, J., Kotla, N.G., Gera, S., Rasala, S., Pandit, A., Rochev, Y.A., Recent Advances in Host-Guest Self-Assembled Cyclodextrin Carriers: Implications for Responsive Drug Delivery and Biomedical Engineering. Adv. Funct. Mater., 2020, DOI: 10.1002/adfm.201909049.

2. Ogoshi, T., Sueto, R., Yoshikoshi, K., Sakata, Y., Akine, S., Yamagishi, T., Host-Guest Complexation of Perethylated Pillar[5]arene with Alkanes in the Crystal State. Angew. Chem. Int. Ed., 2015, 54, 9849-9852.

3. Zhang, D., Cochrane, J.R., Di Pietro, S., Guy, L., Gornitzka, H., Dutasta, J.-P., Martinez, A., "Breathing" Motion of a Modulable Molecular Cavity. Chem. Eur. J., 2017, 23, 6495-6498.

4. Zhang, M., Yan, X., Huang, F., Niu, Z., Gibson, H. W., Stimuli-Responsive Host-Guest Systems Based on the Recognition of Cryptands by Organic Guests. Acc. Chem. Res., 2014, 47, 1995-2005.

5. Loffer, S., Lubben, J., Wuttke, A., Mata, R.A., John, M., Dittrich, B., Clever, G.H., Internal dynamics and guest binding of a sterically overcrowded host. Chem. Sci., 2016, 7, 4676-4684.

6. Kitagawa, H., Kobori, Y., Yamanaka, M., Yoza, K., Kobayashi, K., Encapsulated-guest rotation in a selfassembled heterocapsule directed toward a supramolecular gyroscope. Proc. Natl. Acad. Sci. USA, 2009, 106, 10444-10448.

7. (a) Wu, W.X., Liu, M., Wang, H., Jin, W.J., A Simple Rotor Guest Molecule Mediates the Formation of Cage or Channel Structures of Halogen-Bonding Host Cocrystals. Cryst. Growth Des., 2019, 19, 4378-4384. (b) Adriaenssens, L., Ballester, P., Hydrogen bonded supramolecular capsules with functionalized interiors: the controlled orientation of included guests. Chem. Soc. Rev., 2013, 42, 3261-3277. 
8. Chen, J., Leung, F. K-C., Stuart, M. C.A., Kajinati, T., Fukushima, T., van der Giessen, E., Feringa, B.L., Artificial muscle-like function from hierarchical supramolecular assembly of photoresponsive molecular motors. Nat. Chem., 2018, 10, 132-138.

9. Naumov, P., Prasad-Karothu, D., Ahmed, E., Catalano, L., Commins, P., Mahmoud-Halabi, J., AlHandawi, M. B., Li, L., The Rise of the Dynamic Crystals. J. Am. Chem. Soc., 2020, 142, 1325613272.

10. Roy, I., Stoddart, J.F., Amphidynamic Crystals Key to Artificial Molecular Machines. Trends. Chem., 2019, 7, 627-629.

11. Jin, M., Chung, T.S., Seki, T., Ito, H., Garcia-Garibay, M.A., Phosphorescence Control Mediated by Molecular Rotation and Aurophilic Interactions in Amphidynamic Crystals of 1,4-Bis[tri-(pfluorophenyl)phosphane-gold(I)-ethynyl]benzene. J. Am. Chem. Soc., 2017, 139, 18115-18121.

12. Colin-Molina, A., Jellen, M.J., Rodríguez-Hernández, J., Cifuentes-Quintal, M. E., Barroso, J., Toscano, R.A., Merino, G., Rodríguez-Molina, B., HydrogenBonded Crystalline Molecular Machines with Ultrafast Rotation and Displacive Phase Transitions. Chem. Eur. J., 2020, 26, 11727-11733.

13. Kaleta, J., Bastien, G., Wen, J., Dracinsky, M., Tortorici, E., Cisarova, I., Beale, P.D., Rogers, C.T., Michl, J., Bulk Inclusions of Double Pyridazine Molecular Rotors in Hexagonal Tris( ophenylene)cyclotriphosphazene. J. Org. Chem., 2019, 84, 8449-8467.

14. Paul, M.; Chakraborty, S.; Desiraju, G. R. Sixcomponent molecular solids: ABC[D1(x+y)ExFy]2. J. Am. Chem. Soc. 2018, 140, 2309-2315.

15. Clarke, H.D.; Hickey, M.B.; Moulton, B., Perman, J.A.; Peterson M.L.; Wojtas, L.; Almarsoon, O.; Zaworotko, M.J., Crystal Engineering of Isostructural Quaternary Multicomponent Crystal Forms of Olanzapine. Cryst. Growth Des., 2012, 12, 8, 41944201.

16. Garai, A.; Biradha, K., Binary and Ternary Salts and Cocrystals of 2-(2-(Pyridine-4-yl)vinyl)- $1 \mathrm{H}$ benzimidazole with Aromatic Carboxylic Acids: Solid-State [2 + 2] Reactions, Photoluminescence, and Ammonia-Sensing Properties. Cryst. Growth Des. 2019, 19, 8, 4602-4612.

17. Bhoval, R., Balaraman, A.A., Ghosh, M., Dutta, S., Dey, K.K., Chopra, D., Probing Atomistic Behaviour to Unravel Dielectric Phenomena in Charge Transfer Cocrystals. J. Am. Chem. Soc., 2021, 143, 10241037.
18. Das, S., Mondal, A., Reddy, C.M., Chem. Soc. Rev., 2020, 49, 8878-8896.

19. Yang, Y.D., Ji, X., Lu, Z.-H., Yang, J., Gao, C., Zhang, H., Tang, B.Z., Sessler, J.L., Gong, H.Y., Time-dependent solid-state molecular motion and colour tuning of host-guest systems by organic solvents. Nat. Commun., 2020, 11, 77.

20. Desiraju, G.R., The $\mathrm{C}-\mathrm{H} \cdots \mathrm{O}$ hydrogen bond in crystals: what is it?. Acc. Chem. Res., 1991, 24, 290-296.

21. Hasa, D., Schneider-Rauber, G., Voinovich, D., Jones, W., Cocrystal Formation through Mechanochemistry: from Neat and Liquid-Assisted Grinding to Polymer-Assisted Grinding. Angew. Chem. Int. Ed., 2015, 54, 7371-7375.

22. Jiang, X., Duan, H.B., Jellen, M.J., Chen, Y., Chung, T.S., Liang, Y., Garcia-Garibay, M.A., Thermally Activated Transient Dipoles and Rotational Dynamics of Hydrogen-Bonded and ChargeTransferred Diazabicyclo [2.2.2]Octane Molecular Rotors. J. Am. Chem. Soc., 2019, 141, 1680216809.

23. Vergés-Bach, M., Kitchin, S.J., Harris, K.D.M., Zugic, M., Koh, C.A., Dynamic Properties of the Tetrahydrofuran Clathrate Hydrate, Investigated by Solid State ${ }^{2} \mathrm{H}$ NMR Spectroscopy. J. Phys. Chem. B, 2001, 105, 2699-2706. (b) Nowaczyk, A., Geil, B., Schildmann, S, Bohmer, R., Guest motion in tetrahydrofuran clathrate hydrate studied by deuteron nuclear magnetic resonance. Phys. Rev. B., 2009, 80, 144303.

24. Catalano, L., Pérez-Estrada, S., Wang, H.-H., Ayitou, A. J.-L., Khan, A.I., Terraneo, G., Metrangolo, P., Brown, S., Garcia-Garibay, M.A., Rotational Dynamics of Diazabicyclo[2.2.2]octane in Isomorphous Halogen-Bonded Co-crystals: Entropic and Enthalpic Effects. J. Am. Chem. Soc., 2017, 139, 843-848.

25. Albayrak, C., Zeidler, M.D., Kuchler, R., Kanert, O., Nuclear Magnetic Resonance (NMR) and Quasielastic Neutron Scattering (QNS) of Molecular Liquids. Ber. Bunsen-Ges. Phys. Chem., 1989, 93, 1119. (b) Ripmeester, J.A., Garg, S.K., Davidson, D.W., NMR behavior of the clathrate hydrate of tetrahydrofuran. I. Proton measurements. J. Magn. Reson., 1974, 15, 295. (c) Kirschgen, T.M., Zeidler, M.D., Geil, B., Fujara, F., A deuteron NMR study of the tetrahydrofuran clathrate hydrate Part I: Relaxation pathways. Phys. Chem. Chem. Phys., 2003, 5, 5243-5246.

26. Luger, P.; Buschmann, J., Twist Conformation of Tetrahydrofuran in the Crystal. Angew. Chem., Int. Ed. Engl., 1983, 22, 410. 


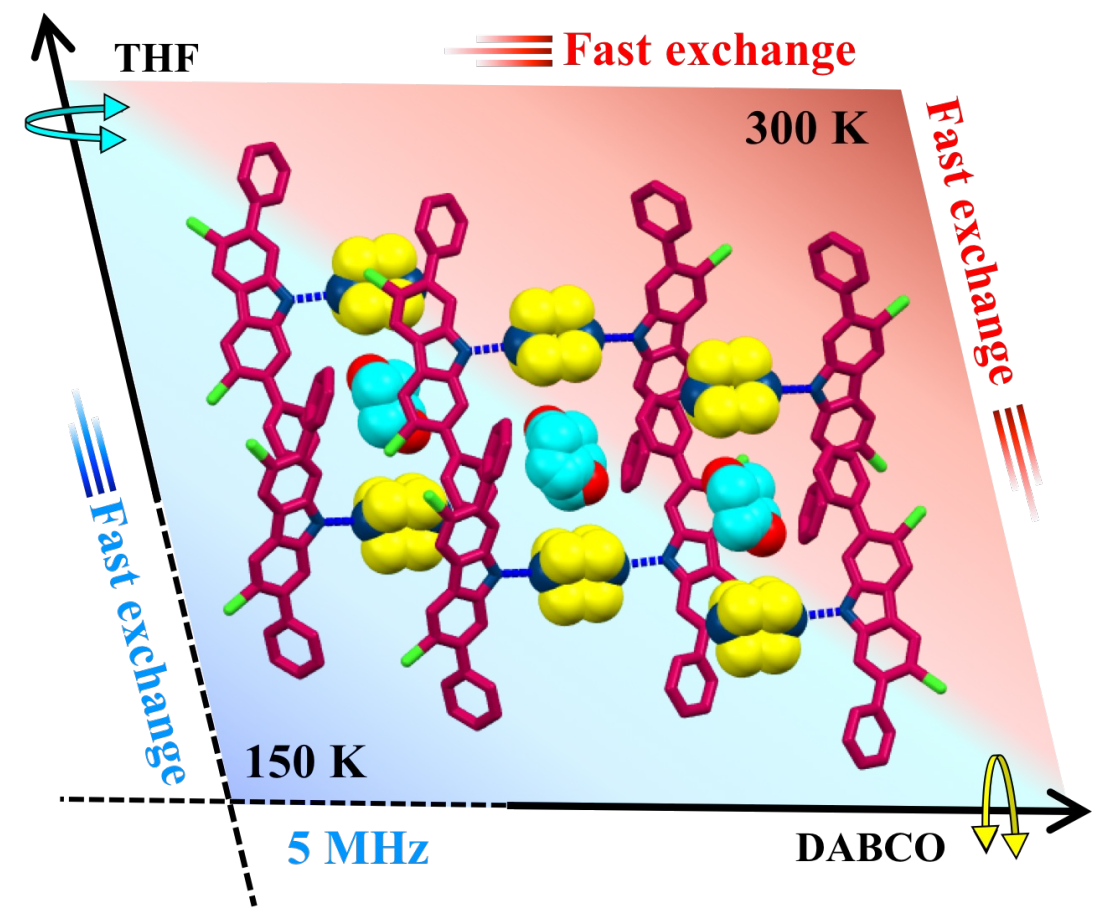

